\title{
Stepping in motor complete spinal cord injured patient with extensor spasticity following intensive gait rehabilitation- case report
}

\begin{abstract}
Introduction: We report the case of a chronic motor complete spinal cord injury (SCI) subject who regained stepping in parallel bars after intensive gait rehabilitation treatment.

Methods: A 32 year old male patient with chronic sensory incomplete SCI (T6, AISA B) with extensor spasticity of both legs, performed over 3 months a locomotor training program 2 years after his initial injury. The training included the use of a robotic system combined with bilateral functional electrical stimulation (FES) of the peroneal nerve for 2 months followed by another month of training on parallel bars with FES.
\end{abstract}

Results: After the three months of training, the patient was able to walk using parallel bars for 2 minutes without FES, orthosis or third person assistance.

Conclusion: A young patient following a complete motor SCI with some preservation of sensory pathways and with extensor spasticity could perform steps following intensive gait rehabilitation.

Keywords: motor complete sci, extensor spasticity, locomotor training, stepping
Volume 3 Issue 2 - 2018

\author{
Narda Murillo, 1,2,3 J Benito-Penalva, ${ }^{1,2,3}$ Hatice \\ Kumru',2,3 \\ 'Neurology and Neurophysiology Unit, Spinal Cord Unit \\ of Fundación Institut Guttmann, Institut Universitari de \\ Neurorehabilitació adscrit a la UAB, Spain \\ 2Univ Autonoma de Barcelona, 08193 Bellaterra (Cerdanyola del \\ Vallès), Spain \\ ${ }^{3}$ Fundació Institut d'Investigació en Ciències de la Salut Germans \\ Trias i Pujol, Spain
}

\begin{abstract}
Correspondence: Hatice Kumru, Hospital de Neurorehabilitació Fundación Institut Guttmann Camí Can Ruti s/n. 08916 Barcelona, Spain, Tel + 349349777 00, Fax+3493497 77 I5, Email haticekumru@yahoo.es
\end{abstract}

Received: February 22, 2017| Published: April 03, 2018
Abbreviations: SCI, spinal cord injury; AIS, american spinal injury association (ASIA) Impairment scale; MAS, modified ashworth scale; FES, functional electric stimulation; VSNs, vestibulospinal neurons; $\mathrm{CPG}$, central pattern generator

\section{Introduction}

For patients who suffer a traumatic spinal cord injury (SCI), the most visible disability is the inability to walk at all or to walk at velocities that don't permit community ambulation. Regaining ambulatory function after a traumatic SCI has been reported at 3\% in initially complete SCI patients AIS-A ${ }^{1,2}$ and is almost impossible in chronic complete motor SCI. ${ }^{3}$ There are just two cases reported, one with chronic complete SCI (AIS-A) (,5 $^{4}$ and one chronic complete motor SCI (AIS-B) ${ }^{7}$ that could perform some steps after locomotor training. Here we describe a patient with a chronic complete motor SCI who was able to do stepping between parallel bars for 2 minutes following an intensive gait training program for 3 months.

\section{Case presentation}

A 32 year-old male patient had a snowboard accident resulting in a traumatic SCI two years prior to commencing this training. On admission, initial neurological examination revealed a motor complete SCI (AIS-B) at the thoracic 6 level. He presented predominantly extensor spasm in both legs with a Modified Ashworth Scale (MAS) score of 3.This allowed him to stand upright for several seconds but without any locomotor capacity. Proprioception of 5-20 degree movements was absent in his toes and knees bilaterally. The neurophysiological assessment showed an absence of motor and sensory evoked potentials in his lower extremities.

Due to the ability of the patient to stand up for a few minutes using extensor spasticity two years after his initial SCI, we decided to initiate an intensive rehabilitation program which included traditional physiotherapy and two months of robotic locomotor training (Lokomat, Hocoma, Switzerland). The training in the Lokomat was done for 30 minutes per day, five days a week, over a two month period, with a velocity of $1.5 \mathrm{~km} / \mathrm{h}, 50 \%$ of bodyweight support, and $100 \%$ guidance force. We added functional electrical stimulation (FES Odstock, Salisbury, UK) on the peroneal nerve to enhance ankle dorsiflexor activity in both legs in order to prevent extensor spasticity from blocking the robotic device during each step cycle.

After two months of FES-assisted robotic gait training, the patient received only FES on the peroneal nerve bilaterally while supporting himself with his arms and performing stepping between parallel bars for one month. After 3 months of gait training, he was able to perform 37 steps using the parallel bars, without an orthosis or third person assistance for a distance of 16 meters in 2 minutes. The average velocity was $0.13 \mathrm{~m} / \mathrm{second}$ with a stride length $0.84 \mathrm{~m}$.

\section{Discussion}

We report the case of a patient with chronic complete motor SCI with severe extensor spasticity in both legs that allows him to stand and to perform stepping for a few minutes between parallel bars after intensive locomotor training. Here we discuss the role of different central nervous pathways on stepping and/or gait following chronic complete motor SCI.

In the literature, there are only a few case reports regarding chronic severe SCI realizing some form of locomotor function. Wernig \& Müller ${ }^{6}$ reported a 32 years old patient (T6, AIS-C) with absent voluntary activity in both legs except in TA unilaterally and severe bilateral extensor spasticity who was capable of walking $40 \mathrm{~m}$ with a walker following 10 years of individual training. Behrman \& Harkema $^{4}$ described the case of a 20 years old person with a complete 
chronic SCI (T5, AIS-A) who could produce 3 to 10 consecutive steps with manual assistance to one leg on the treadmill after gait training. Manella et al. ${ }^{5}$ reported a 33 years old individual with chronic SCI (T7, AIS-A) who developed over ground walking ability following intensive physical therapy and robotic locomotor training. Murillo et al. ${ }^{7}$ reported a single case who was 15 years old with a chronic motor complete SCI (T6, AIS B) and severe bilateral extensor spasticity that, at the end of training, the patient was able to cover a distance of 200 meters with a walker and assistance pulling the walker with each step to help her accomplish effective over ground stepping.

The severity of lesion, severe extensor spasticity and youth were common characteristics of the last case and this reported case, however, the current case being reported did not require an orthesis or person to provide assistance for stepping using the parallel bars.

In all of these case reports we can observe commons characteristics: the patients were young (3, 20, 33 and 15 years old respectively), all had chronic lesions, three of them presented extensor spasticity, and they all participated in an intensive, specific locomotion training program

Age is an important factor for the development of neuronal plasticity ${ }^{8}$ which could contribute to the learning of locomotion in the spinal cord. Furthermore, Oleason CV et al. ${ }^{9}$ observed a correlation between an initial pinprick sensation (AIS B) and age under 50 years that favored a positive prognosis for walking in people living with a SCI.

Three other previously published patients presented with similar bilateral spastic extensor patterns as our current patient. ${ }^{5-7}$ Extensor spasticity is defined by Steldt RE \& Schmit BD. ${ }^{10}$ as a multipoint reflex response to bilateral hip extension, and is characterized by hip flexion, knee extension, and ankle extension torques. it is a recurring feature in all these reported cases and it could be an identifying characteristic which could predict the success of an intensive locomotion training program.. This extensor spasticity would be conditioned to the location of the lesion, possibly because the ventral part of the spinal cord is less damaged, how it is observed in studies in animals such as rats or cats, in which the vestibulospinal tract is described as responsible for activating the antigravitational extensor muscles during the stance phase of the gait cycle. ${ }^{11,12}$ It is possible that feedback from the otoliths, from peripheral receptors, or centrally from interneuron signaling information about the Spinal Central Pattern Generator (CPG) will modulate the activity of the vestibulospinal neurons (VSNs) to ensure that their maximum output occurs at times in the step cycle at which there is the greatest need for antigravity activity. ${ }^{11}$ Along with the vestibulospinal pathways, the reticulospinal pathways are also implicated in the step-by-step control of locomotion. Given their widespread projections, these cells may participate in the coordination between limbs as well as in weight support directly or as an integrative relay between the cortex and spinal cord. ${ }^{13}$

Besides these pathways, it is clear that on lesion type AIS B, patients have a certain grade of sensation that means that they have some dorsal pathways preserved. In rats, it has been observed that lesions at the Rubrospinal nucleus cause persistent deficits during over ground and skilled locomotion. ${ }^{13}$ Hence preservation of this nucleus is a conceivable condition needed to initiate locomotion.

In addition to young age, some sensory preservation and extensor spasticity, intensive and specific training might be the other important factor for recuperation of stepping as it promotes neuronal plasticity by increasing the learning of the neural circuits involved in locomotion. ${ }^{14}$ According to Smith $\mathrm{AC} \&$ Knikou M, ${ }^{15}$ locomotor training promotes functional neuronal reorganization. A major driver for neuronal reorganization after locomotor training is reinforcement of activity-dependent sensory feedback from receptors that can adjust the operation of the CPG.

\section{Conclusion}

The spinal cord may improve some of its locomotor function following gait rehabilitation in younger patients with chronic complete motor SCI with sensory pathway preservation and the presence of extensor spasticity. It is possible that extensor spasticity activity facilitates standing in young patients and together with some preserved ventral and dorsal pathways, could facilitate stepping depending on the intensity of locomotor training.

\section{Patient consent Form}

Yes

\section{Acknowledgements}

None.

\section{Conflict of interest}

The authors declare no conflict of interest.

\section{References}

1. Marino RJ, Barros T, Biering SF, et al. International standards for neurological classification of spinal cord injury. J Spinal Cord Med. 2003;26Suppl 1:S50-56.

2. Marino RJ, Burns S, Graves DE, et al. Upper- and lower-extremity motor recovery after traumatic cervical spinal cord injury: an update from the national spinal cord injury database. Arch Phys Med Rehabil. 2011;92(3):369-75

3. Yang JF, Musselman KE. Training to achieve over ground walking after spinal cord injury: a review of who, what, when, and how. J Spinal Cord Med. 2012;35(5):293-304.

4. Behrman AL, Harkema, SJ. Locomotor Training after Human Spinal Cord Injury: A Series of Case Studies. Phys Ther. 2000;80(7):688-700.

5. Manella KJ, Torres J, Field FEC. Restoration of walking function in an individual with chronic complete (AIS A) spinal cord injury. J Rehabil Med. 2010;42:795-798.

6. Wernig A, Muller S. Laufband locomotion with body weight suppor improvedwalking in persons with severe spinal cord injuries. Paraplegia. 1992;30(4):229-238.

7. Murillo N, Kumru H, Opisso E, et al. Recovery of assisted overground stepping in a patient with chronic motor complete spinal cord injury: a case report. NeuroRehabilitation. 2012;31(4):401-7.

8. Jaerve A, Schiwy N, Schmitz C, et al. Differential effect of aging on axon sprouting and regenerative growth in spinal cord injury. Exp Neurol. 2011;231(2):284-94.

9. Oleson CV, Marino RJ, Leiby BE, et al. Influence of Age Alone, and Age Combined With Pinprick, on Recovery of Walking Function in Motor Complete, Sensory Incomplete Spinal Cord Injury. Arch Phys Med 
Rehabil. 2016;97(10):1635-41.

10. Steldt RE, Schmit BD. Modulation of coordinated muscle activity during imposed sinusoidal hip movements in human spinal cord injury. Journal of Neurophysiology. 2004;92(2):673-85.

11. Matsuyama K, Drew T.Vestibulospinal and reticulospinal neuronal activity during locomotion in the intact cat. I. Walking on a level surface. J Neurophysiol. 2000;84(5):2237-56.

12. Orlovsky GN. The effect of different descending systems on flexor and extensor activity during locomotion. Brain Res. 1972;40:359-371.
13. Rossignol S, Barrière $\mathrm{G}$, Alluin $\mathrm{O}$, et al. Re-expression of locomotor function after partial spinal cord injury. Physiology (Bethesda). 2009;24:127-39.

14. Grasso R, Ivanenko YP, Zago M, et al. Distributed plasticity of locomotor pattern generators in spinal cord injured patients. Brain. 2004;127(Pt 5):1019-34.

15. Smith AC, Knikou M. A Review on locomotor training after spinal cord injury: reorganization of spinal neuronal circuits and recovery of motor function. Neural Plast. 2016:1216258. 